\title{
"HE CRECIDO AQUÍ, ES DIFERENTE". EXPERIENCIAS Y TRAYECTORIAS VITALES DE LOS/AS DESCENDIENTES DE MIGRANTES CHINOS EN ESPAÑA
}

\author{
“I GREW UP HERE, IT'S DIFFERENT". \\ EXPERIENCES AND VITAL PATHS OF THE \\ CHINESE MIGRANTS' DESCENDANTS IN SPAIN
}

Laura Lamas ABraira*

\begin{abstract}
Resumen: Este artículo explora las experiencias y trayectorias de los/as descendientes de migrantes chinos en España a partir de una perspectiva de curso vital: desde su infancia temprana, hasta su condición actual como jóvenes adultos/as. A través de una investigación etnográfica y en base a los testimonios en primera persona de los/as informantes, se analiza el posicionamiento e interacción de estos en su entorno familiar y en el contexto social más amplio, así como los factores que los condicionan. Familia, cuidados y diversas nociones de la 'diferencia' vertebran su relato aunando recuerdos, percepciones y expectativas en tiempo pasado, presente y futuro en las que referencias a lo 'español' y lo 'chino' son
\end{abstract}

* Este artículo cuenta con el apoyo de: Proyecto I + D "Nuevos desarrollos socioculturales, políticos y económicos de Asia Oriental en el contexto global” (PID2019-107861GB-I00, MINECO/FEDER, UE), Beca doctoral de la Fundación Chiang Ching-Kuo (2018/2019) y Beca Doctoral del Programa Confucius China Studies (2016/18-2017/18).

MIGRACIONES 52 (2021). ISSN: 2341-0833, DOI: 10.14422/mig.i52.y2021.005 117-146 
constantes. En base a la información obtenida, se subraya la necesidad de abogar por estudios que aporten una visión no fragmentada de las trayectorias de los/as descendientes de familias migrantes y un mayor énfasis en la noción de interconexión.

Palabras clave: descendientes migrantes; curso vital; jóvenes adultos; España; China.

Abstract: This paper explores the experiences and life paths of Chinese migrant's descendants in Spain from a life course perspective: from their early years to their actual condition as young adults. Using an ethnographic approach and based on informants' first-person accounts, it analyses their positioning and interaction within their families and broader social context, as well as the conditioning factors. Family, care and diverse notions of 'difference' vertebrates their discourse, pooling together memories, perceptions and expectations in past, present and future time, in which references to 'the Spanish' and 'the Chinese' are constant. Based on collected data, the desirability of carrying on studies that emphasizes the notion of interconnection and provide a non-fragmented view of the trajectories of the descendants of migrant families is underlined.

Key words: migrants' descendants; life path; young-adults; Spain, China.

\section{INTRODUCCIÓN}

La migración continuada y cuantitativamente significativa de China a España se inició en la década de los 80, experimentando su mayor crecimiento entre los años 1995-2000. Este flujo migratorio procede, en su amplia mayoría, del condado de Qingtian y la vecina ciudad de Wenzhou —ambos en la provincia de Zhejiang- y se caracteriza por su origen rural, su carácter familiar, su actividad empresarial y la dispersión geográfica derivada de esta (Beltrán Antolín, 2003, 2006; Sáiz López, 2005, 2012), aunque el origen geográfico y sociocultural de la migración china en España ha empezado a diversificarse en el s.XXI ${ }^{1}$. Sin embargo, en las décadas

1 Incluyendo un flujo reciente de migrantes llegados desde grandes ciudades chinas, como Beijing, Shanghai o Guangzhou y provincias como Fujian, en el sur, y Shangdong, Liaoning, Heilongjiang y Jilin, en el norte. Estas personas, en 
precedentes la migración de origen chino en España compartía un mismo patrón migratorio y de asentamiento. Con una legislación migratoria ajena al sistema de cupos en aquel momento, el ciclo comenzaba con una persona migrante-hombre o mujer dependiendo de los contactos existentes (Sáiz López, 2005) - llegando a España para trabajar como asalariada en el negocio familiar de sus parientes o paisanos/as. Su trabajo le permitía pagar las deudas relacionadas con el viaje a España y sus formalidades así como, junto a un estilo de vida muy austero, ahorrar para poder abrir su propio negocio más tarde, habiendo ya adquirido los conocimientos necesarios para sacar adelante el mismo y posibilitando la continuidad de la cadena migratoria (Beltrán Antolín, 2003, 2006).

El nicho económico mayoritario en la década de los ochenta eran los restaurantes chinos, empezando a diversificar (tiendas multiprecio y de conveniencia, zapaterías y venta al por mayor) a mediados de los noventa (Beltrán Antolín, 2003, 2006; Sáiz López, 2005). Aunque la migración desde las áreas de Qingtian y Wenzhou hacia España comprende a personas de todas las clases sociales (Beltrán Antolín, 2003, 2006; Sáiz López, 2005) constituyendo a finales del s.XX la migración hacia Europa ${ }^{2}$ un imperativo social en la zona (Masdeu Torruella, 2014a), existe una mayoría de personas de origen rural y humilde, con un bajo nivel de educación formal. No obstante, el propósito de movilidad social ha sido alcanzado con éxito en la mayoría de estas familias, que vuelcan sus recursos en las nuevas generaciones.

Dada la corta historia migratoria, de manera generalizada, no ha sido sino recientemente que los/as jóvenes descendientes de migrantes chinos en España han comenzado a alcanzar la mayoría de edad, constituyendo la primera generación ${ }^{3}$ de su familia que ha sido, total o parcialmente, socializada en España. Este artículo toma a esta generación como protagonista y, desde una perspectiva

contraste con los/as migrantes de Zhejiang tienden a ser de origen urbano y con un nivel educacional mayor, trabajan como asalariadas y no tienen un proyecto de asentamiento en España (Beltrán Antolín, 2006; Sáiz López, 2005, 2012).

2 Es habitual que estas familias cuenten con miembros de la familia extensa distribuidos en diferentes países europeos y, muy especialmente, en Italia.

3 Entendiendo en este texto 'generación' como ambos, un grupo de aproximadamente la misma edad que experimenta los mismos eventos históricos en un determinado estadio vital, así como un mismo posicionamiento en el rango genealógico familiar (Foner, 2009b). 
de curso vital, busca dar respuesta a las siguientes preguntas: ¿qué experiencias caracterizan la infancia y posteriores etapas vitales de los/as informantes?, ¿cómo éstas se interrelacionan entre sí?, ¿cómo afecta su condición de descendientes de migrantes chinos en España a sus vivencias y trayectorias?, ¿cómo el carácter transnacional de sus familias?, ¿responden éstas a patrones comunes?

Para ello, esta contribución se estructura como sigue. Primero, se presenta el marco teórico y se introduce la metodología utilizada en la investigación. A continuación, se presenta la trayectoria de los/ as informantes utilizando para ello marcas temporales en relación a sus diferentes etapas vitales, en torno a las cuales se organiza la discusión de los temas subyacentes. Para terminar, se incluyen algunas reflexiones finales a modo de conclusión.

\section{MARCO TEÓRICO}

A lo largo de la última década, desde la academia se ha subrayado la necesidad de superar la perspectiva adulto-céntrica que domina el estudio de las migraciones y redirigir el foco hacia los/as menores de edad y sus experiencias (Gardner, 2012; Ni Laoire, 2011; White et al., 2011; Dobson, 2009). Ello conlleva incorporar sus voces y puntos de vista en tiempo presente, pero también en retrospectiva (Phoenix y Seu, 2013; Phoenix y Bauer, 2012; Olwig, 1999), permitiendo así incorporar una perspectiva de curso vital que evite focalizar en momentos o etapas específicas y abogar así por una visión de sus realidades más coherente y menos fragmentada.

Las investigaciones sobre migración y familias chinas en las que los menores tienen un rol protagonista, en su mayoría, se centran en arreglos de cuidado trasnacional durante la infancia temprana. Estas arreglos engloban prácticas de 'abuelazgo' transnacional (transnational grandparenting), en las cuales los abuelos/as viajan a otro país para ejercer de cuidadores (Da, 2003; Lie, 2010, Neysmith y Zhou, 2013; Zhou, 2013) y la práctica de enviar los/as menores al país de origen de sus progenitores, conocida globalmente como 'niños/as enviados de vuelta' (sent-back children) y que en el caso

4 Pese a que a menudo los niños/as han nacido en otro país y por tanto no implica un regreso físico como tal. 
chino se designa mayoritariamente como 'bebés satélite' (satellite babies), dado la temprana edad a la que son enviados a China (Bohr y Tse, 2009; Wong, 2015), para posteriormente reunirse con la familia nuclear en el país de destino de la migración.

En esta misma línea, encontramos la práctica comúnmente denominada como 'niños/as dejados atrás' (left-behind children), en alusión a aquellos/as menores que permanecen en el país de origen mientras sus progenitores emprenden el proyecto migratorio. Pese a que abundan los estudios sobre el tema en lo relacionado con la emigración interna en China (Biao, 2007; Chang, Dong y MacPhail, 2011; Guang et al., 2017; Lei, Liu y Hill, 2017; Lu, 2012; Wen y Li, 2012) y en otros contextos transnacionales (Antman, 2012; Pantea, 2011; Parreñas, 2005; Perreira y Ornelas, 2011; Suárez-Orozco, Bang y Ha, 2012; Tienda y Haskins, 2011; Tymczuk, 2011), en los cuales prima el discurso del abandono y la familia disfuncional (Pantea, 2011), apenas existe información sobre aquellos/as menores que permanecen en China mientras sus progenitores emigran a otros países (Morooka y Liang, 2009).

Pantea (2011) critica la falta de estudios centrados en adolescentes y jóvenes en comparación con el número creciente de investigaciones sobre menores de corta edad. Los estudios que tienen como protagonistas a jóvenes descendientes de migrantes ponen el foco sobre el mantenimiento de la cultura e idioma de la sociedad de origen de la familia (Said y Zhu, 2017; Wei, 2011), su identidad (Kibria, 1997; Louie, 2006; Raffaetà et al., 2015; Said y Zhu, 2017; Sigad y Eisikovits, 2010), sus vínculos y experiencias transnacionales (Bartley y Spoonley, 2008; Gardner y Mand, 2012; Haikkola, 2011; Kim, 2009; Levitt, 2009), su inserción laboral (Gaspar, 2019; Yiu, 2013) y las relaciones intergeneracionales, incluyendo los procesos de reunificación familiar (Foner, 2009a; Foner y Dreby, 2011; Menjívar 2006; Artico 2003; Suárez-Orozco, Todorova y Louie, 2002) y apuntando al rol central de las relaciones familiares en "dar forma a las transiciones de los jóvenes y las trayectorias migrantes" (Punch, 2014, pp. 262).

Asimismo, existe un amplio cuerpo de estudios académicos centrados en el concepto de integración en la llamada sociedad de destino y que apuntan a una generalizada disonancia generacional en base a una diferente socialización (Marsden, 2005; Pavez Soto, 2012; Waters y Sykes, 2009; Wu y Chao, 2011; Zhou, 2009),con una creciente importancia de la perspectiva de la teoría de la 
'asimilación segmentada' (Portes, 1997, 2018; Portes y Rumbaut, 2001; Zhou, 1997). Dicha teoría incorpora al análisis tanto elementos estructurales como culturales, ofreciendo un importante avance con respecto a las teorías anteriores. Sin embargo, la fijación por el concepto 'integración', así como el de aculturación ${ }^{5}$, y la necesidad de establecer categorías - tanto dicotómicas como de gradación - para poder elaborar una teoría coherente en su aplicación generalizada, nublan e invisibilizan realidades intermedias, relacionales y cambiantes que a menudo caracterizan las experiencias y trayectorias de los/as sujetos de estudio de la investigación etnográfica.

En el contexto español no existen investigaciones centradas en las experiencias y trayectorias específicas de los/as menores descendientes de migrantes chinos. Sin embargo, sus realidades se han visto parcialmente reflejadas a través de otros estudios centrados en la unidad familiar (Beltrán Antolín, 2003; Masdeu Torruella, 2014; Nieto, 2007; Sáiz López, 2012). A menudo, a fin de que las parejas de jóvenes migrantes de origen chino puedan centrarse en la esfera productiva, de cara a mejorar las expectativas de futuro de las diferentes generaciones familiares, estas deben decidir entre contratar o transnacionalizar el trabajo reproductivo (Sáiz López, 2012). Así, es habitual que las personas que conforman la generación menor hayan sido parcialmente criadas en China, al cuidado de miembros de la familia extensa, y posteriormente reunificadas con la familia nuclear en España. En menor medida, también abuelos/as se desplazan a España para ejercer de cuidadores.

Asimismo, en los últimos años la generación de los/as jóvenes adultos se ha convertido en protagonista de investigaciones, estando estas directamente relacionadas con sus experiencias de movilidad (Masdeu Torruella, 2019; Masdeu Torruella y Sáiz López, 2019), su construcción identitaria (Robles-Llana, 2018) o su inserción laboral (Yiu, 2013). Pese al creciente interés académico por esta generación, estas investigaciones carecen en su mayoría de una perspectiva de curso vital y se centran principalmente en ámbitos y/o momentos

5 Proceso de asimilación de influencias culturales de la sociedad del país de destino de la migración familiar por parte de los migrantes y/o sus descendientes. Para un mayor énfasis en el tema y una categorización en base a gradaciones (aculturación consonante, aculturación selectiva y aculturación disonante), ver Portes (1997, 2018). 
específicos de sus vidas. Buscando superar esta limitación, este artículo explora las experiencias y trayectorias de los/as informantes a través de su ciclo vital: desde su nacimiento hasta su condición actual como jóvenes adultos/as.

\section{METODOLOGÍA}

Este estudio forma parte de un proyecto de investigación de mayor envergadura llevado a cabo entre 2014 y 2019 bajo la forma de una etnografía multisituada, con un total de 6 meses de trabajo de campo en España y 13 en China. La información a la que se hace referencia en este artículo ha sido recogida mediante 21 entrevistas semi-estructuradas y observación participante. La muestra se compone de descendientes de migrantes que pertenecen a familias transnacionales chinas cuya región de origen es la provincia de Zhejiang y en las cuales, en la actualidad, la residencia habitual de las personas que forman parte de la familia nuclear se encuentra en España. Asimismo, se denomina 'jóvenes adultos' a los/as informantes entre 18 y 31 años y sin descendencia en el momento de la entrevista, prevaleciendo así su rol familiar de hijo/a. ${ }^{6}$

El muestreo se ha realizado mediante la práctica de bola de nieve e incorpora tres tipos de trayectorias (Fresnoza Flot, 2018) de las personas informantes: 5 nacidas y criadas en España, 10 nacidas en Europa (8 en España, 1 en Italia y 1 en Portugal) que fueron enviadas a China y posteriormente se reunieron con la familia nuclear en España y 6 informantes que permanecieron en China mientras sus progrenitores emigraban y, años más tarde, se reunieron con ellos en España mediante procesos de reagrupación familiar. Por tanto, de las 21 personas informantes, 16 de ellas pasaron una parte de su infancia en China. En aras de preservar su anonimato, los nombres utilizados en el texto para referirse a los informantes son pseudónimos.

6 Asimismo, el término 'adolescente' se usa en este artículo para hacer referencia a aquellas personas en edades comprendidas entre 12 y 18 años y 'niño/a' para aquellas personas menores de 12 años. Dicha división se basa en los sistemas educativos chino y español, los cuales separan el nivel de educación primaria y secundaria a la edad de 12 años, sirviendo a su vez de marcador social. 
Las entrevistas han sido realizadas bien en España o en China, siendo el español el idioma utilizado. Estas han sido transcritas y posteriormente codificadas y analizadas conjuntamente con las notas de campo, utilizando para ello el método de Análisis temático inductivo (Braun y Clarke, 2012) con la ayuda del programa de análisis cualitativo Nvivo 11, a través del cual se han identificado patrones temáticos comunes que han dado forma a la estructura de la discusión que sigue.

\section{RESULTADOS}

La infancia temprana (aprox. 0-6 años)

Manuel (25 años): Nos íbamos por ahí con mis abuelos, nos daban de comer, nos cuidaban, nos duchaban y si estaban mis tíos me iba a la montaña un poco con ellos (...) [Infancia en China]

Daquan (29 años): bueno (...) es que es un poco raro, durante el primer año no me cuidaron mis padres, me cuidó una pareja española porque ellos no podían. [Infancia en España]

Sonia (21 años): Mi abuela es como mi madre. Me crié con ellos. [Infancia en España]

Esta primera etapa es especialmente diferencial en lo que confiere a los diferentes grupos dentro de la muestra. Sus experiencias se ven mediadas principalmente por el factor espacial: mientras unos/as nacían y crecían en España, otros/as nacían en Europa y crecían en China y otros/as nacían y crecían en China. En concordancia con la citada literatura sobre los/as 'bebés satélite', la edad a la que las personas entrevistadas fueron enviadas a China fue muy temprana, contando, salvo una excepción, con menos de dos años de edad. En este sentido, y dada la corta edad de los/as informantes en aquel momento, las experiencias de aquellas personas nacidas en China y aquellas que llegaron como 'bebés satélite', convergen. Para estos/ as menores parcialmente criados en China, la generación de los abuelos/as asumió el cuidado diario, situación que es generalizada en el país (Chen, Liu y Mair, 2011), si bien el papel de las tías es a menudo también importante.

El rol de cuidadora principal recaía en las abuelas - con la excepción de una de las familias- y a menudo los abuelos 
continuaban trabajando fuera del hogar ${ }^{7}$. Cabe destacar también que los arreglos de cuidado eran flexibles. Así, en esta pequeña muestra, encontramos dos casos en los cuales menores estaban al cargo de la generación de los abuelos/as pese a que no había relación de parentesco directo con estos. Esto es, en dos de los casos los menores vivían con los abuelos/as de sus primos/as, pese a que no existía consanguineidad entre ellos. Estos primos/as a menudo también vivían separados de sus progenitores, quienes habían emigrado a España u otro país europeo.

La narración de sus experiencias es poco detallada. Sin embargo, incorpora elementos similares: una vida feliz y ociosa en el pueblo o la montaña con miembros de la familia extensa. Así, sus testimonios cuestionan frontalmente la idea de la etapa en China como infancia desafortunada y tiempo de carencias, de menores pseudo abandonados/as (Pantea, 2011) y llaman a relativizar y situar en contexto el periodo de separación. Raras veces recuerdan visitas de sus madres o padres. Cabe decir que, aunque en su mayoría recibieron la visita de uno de sus progenitores al menos una vez durante su infancia, no en todos los casos éstos pudieron viajar a China para visitarles. Asimismo, dada la temprana edad a la que se separaron de los mismos y, por tanto, la ausencia de fuertes vínculos afectivos, no reportan haberles echado de menos durante esta etapa. Salvo una excepción, una informante nacida en China para quien el movimiento migratorio paterno ocurrió a la edad de tres años y, por tanto, era ya consciente de la situación y narra la angustia ante la próxima separación durante una visita que tuvo lugar a la edad aproximada de cinco años.

En lo referente a aquellos/as informantes que permanecieron en España, la casuística en cuanto a arreglos de cuidado es diversa y no excluyente. Así, encontramos casos en los que la generación de los abuelos/as viajó a España en los meses próximos al nacimiento de un nuevo miembro de la familia o en diferentes ocasiones a lo largo de los años. En dos de los casos dicha generación residía previamente en España ${ }^{8}$ y ejerció el rol de cuidador principal.

7 La edad de jubilación en China es menor para las mujeres que para los hombres, enfatizando el rol de cuidadoras de las abuelas.

8 En estas dos familias, la excepción entre la muestra, fueron los abuelos/ as y no la madre/padres los primeros en emigrar, sin embargo, sus hijos/as (los progenitores de nuestros informantes) no fueron socializados en España y migraron como jóvenes adultos. 
Para una de estas familias, el domicilio de la familia nuclear y el de los abuelos/as se situaba a apenas unos minutos de distancia, facilitando el proceso. En el otro caso,el abuelo, ya viudo, residía en otra ciudad dentro del estado español y se mudó durante un tiempo a la ciudad en la que su hija y yerno vivían para ejercer de cuidador principal de los menores, uno de los cuales (el mayor) había sido enviado previamente a China.

No obstante, la ayuda por parte de la familia extensa no siempre es una opción o esta sirve solamente como arreglo temporal. Así, tres de las familias de los/as informantes subcontrataron el cuidado, en este caso, a personas de origen español. Una de ellas, una chica joven sin descendencia, que realizaba labores de cuidado durante el día, acudiendo al hogar familiar. En otro caso, la cuidadora principal se trataba de una mujer de edad avanzada que cuidaba de sus propios nietos/as y que incorporó al menor en su hogar mediante un proceso de circulación informal que se prolongó hasta la adolescencia. Finalmente, uno de los informantes pasó su primer año de vida siendo cuidado por una familia española en el hogar de éstos, para más tarde regresar al hogar de su familia natal y convertirse su madre en su cuidadora principal.

La niñez (aprox. 6-12 años)

Xingfu (27 años): Supongo que sí que habría visto alguna foto suya, pero yo no los conocía de nada. Ya me dirás tú qué significa una foto para un niño de 5 años.

Dora (22 años): Cuando me fui, allí sí [a España], lloré mucho (...) me voy, me tengo que separar de mis abuelos, eh, por eso lloraba mucho.

Feifei: En mi infancia, mis padres estaban siempre ocupados y no se ocupaban mucho de mí.

Aquellas personas que pasaron sus primeros años de vida en China, ya sea habiendo nacido allí o llegando como 'bebés satélite', se mudaron a España en torno a los 4-7 años de edad para ser escolarizadas, salvo en dos excepciones, en las que lo hicieron a los 12 y 13 años respectivamente. Solo algunos/as sabían con antelación que viajarían a España y ello despertó cierta curiosidad y una actitud más positiva ante el viaje. Al resto, la partida les pilló de imprevisto y aunque no querían, acabaron viajando a España. Así, su posición fue completamente ignorada en la toma de la decisión, tal y como 
ocurre en otras familias migrantes (Dreby, 2007; Hoang, Lam, Yeoh y Graham, 2015; Orellana, Thorne, Chee y Lam, 2001). Una minoría de los/as informantes llegaron a España acompañados por sus cuidadores habituales. Para el resto, uno de sus progenitores viajó a China para acompañarles y en uno de los casos, la informante no recuerda si fue su madre o su padre quien fue a recogerla.

Varios/as informantes recuerdan haber llorado mucho durante el viaje y sus primeros días en España. Querían volver a China con sus cuidadores y mencionan que, debido al alto coste de las llamadas en aquellos años, éstas eran escasas. Por otro lado, algunos/as informantes apuntan a que sus madres y padres les resultaban extraños y aunque les habían enviado fotos para que pudieran reconocerles, no eran suficiente, como se deduce del comentario de uno de los informantes, citado al inicio de este apartado. Relatan haber sentido tensión al reunirse con ellos, sentimiento que a menudo sirve para describir los procesos de reunificación familiar en familias migrantes (Bonizzoni, 2015; Menjívar, 2006).

El verbo 'acostumbrarse' es a menudo evocado. Leinaweaver (2007), en su investigación sobre la circulación informal de menores en los Andes, refiere a este mismo concepto, acostumbrarse, como "una forma productiva de pensar en la transformación en el comportamiento, tratamiento, confort y sentimiento que finalmente resulta en familia" (Leinaweaver, 2007, p. 169), en referencia a la relación del/a menor con ambos, personas y lugares a través del tiempo. Así, en este caso, la movilidad internacional implica una experiencia física y emocional, en el entorno más próximo, de la misma familia, así como también en el contexto social más amplio.

La reunificación con hermanos/as no ha sido descrita como conflictiva, sino al contrario, encontrando en ellos/as un punto de apoyo. Del mismo modo, ninguno/a de los/as informantes tuvo que conocer a la nueva pareja de su madre/padre, ya que estos continuaban juntos, por lo que no confrontaron ninguna situación especialmente conflictiva en el seno familiar (Bauer, 2016; Menjivar, 2006; Phoenix y Bauer, 2012). Asimismo, no ha habido referencias a intentos de desafío a la autoridad familiar (Artico, 2003; Menjívar, 2006) durante esta fase, aunque cabe recordar que, salvo en dos excepciones, eran muy pequeños/as cuando llegaron a España.

En contraste con la idealización de la etapa anterior, de su infancia en China, y la ausencia de sentimientos de falta durante la misma, en esta, la separación de sus cuidadores habituales hasta ese momento 
y la angustia derivada de este hecho es generalizada entre los/as informantes (Suárez-Orozco, Bang y Ha, 2012), aunque acotada en el tiempo. Cabe destacar que dicha sensación está mediada por la falta de recursos estructurales y económicos para dotar las relaciones transnacionales de continuidad, dado los altos costes del transporte y comunicaciones internacionales del momento?.

Los sentimientos de tristeza, frustración e impotencia no duraron mucho tiempo, o al menos así lo recuerdan a día de hoy. Salvo una excepción, un chico para el que la idea de regreso a China perduró en el tiempo. No obstante, su narración pone de manifiesto que su descontento emanaba de su interacción social más amplia, no del ámbito familiar. Las personas entrevistadas que pasaron parte de su infancia en China recuerdan las dificultades iniciales para adaptarse al nuevo entorno, especialmente debido a que no hablaban español, aunque no obstante, esta circunstancia es también compartida por algunas de las personas nacidas y criadas en España, para quienes el idioma español apenas formaba parte de su día a día antes de la escolarización. Existe una percepción compartida por toda la muestra -independientemente de su crianza parcial en China o no- de la entrada en el colegio como el momento en el que la idea de la diferencia, aunque por aquel entonces no siempre entendida o articulada como tal, aparece en sus vidas a raíz de la interacción directa y continuada con los demás alumnos/as y las dinámicas escolares.

Así, en esta etapa, los/as personas informantes de los tres grupos - nacidas en China, nacidas en Europa y enviadas a China y aquellas nacidas y criadas en España- dado que tenían más de 4 años fueron escolarizadas y, por tanto, gran parte del día lo pasaban en el colegio. El negocio familiar - restaurante o tienda- a menudo aparece como el lugar diario de reunión para los distintos miembros de la familia (Pavez Soto, 2012b) donde los/as informantes jugaban y realizaban las tareas escolares. No obstante, las posibilidades de interacción e intercambio generacional no eran generalizadas y la carga de trabajo prevalecía.

9 De la comparativa de las experiencias de estos/as jóvenes adultos con otro grupo de informantes, en la actualidad adolescentes, se ha documentado una correlación entre una valoración más positiva del momento de la reunificación y la posibilidad de mantener comunicaciones transnacionales frecuentes con los diversos miembros de la familia, tanto antes como después de la reagrupación en España (Lamas Abraira, 2021). 
Asimismo, durante esta etapa se documentan arreglos ocasionales o temporales que envolvían miembros de la familia extensa, hermanos/as mayores, amigos/as de la familia y, en menor medida, cuidadoras contratadas. Existe en su relato en tiempo presente sobre esta etapa una fuerte dimensión crítica en relación a la priorización del trabajo productivo ante el reproductivo, así como ante la falta de interacción diaria y/o tiempo de calidad en familia - pese a la posible co-presencia y pernoctación bajo el mismo techo- que está presente de forma generalizada en todos/as los/as informantes, independientemente de su crianza temprana en China o en España.

Pese a que en esta sección se ha dedicado una parte importante del texto a describir las experiencias relacionadas con el cambio de contexto - de China a España- de los/as informantes, en tanto que constituyentes de una diferencia con respecto a la muestra. El espacio y atención dedicados no es directamente proporcional al dedicado al mismo por los/as informantes durante las entrevistas. Ello no quiere decir que carezcan de importancia, al contrario, son extremadamente importantes. Estas experiencias sirven como anclaje imprescindible en sus trayectorias vitales e implican una fuerte carga emocional ${ }^{10}$. No obstante, en sus relatos cobran fuerza en una acotación temporal concreta y muy limitada en lo que se refiere a sus recuerdos y, en cambio, otras circunstancias que caracterizan su vida una vez ya en España, como una articulación incipiente de la diferencia en el entorno social más amplio, el negocio familiar y la calidad de la relación con sus progenitores - todas ellas circunstancias compartidas con aquellas personas informantes nacidas y criadas en España, en base a su condición de familias migrantes - se tornan las protagonistas de sus vivencias durante esta etapa.

La adolescencia (aprox. 12-18 años)

Dandan: En la adolescencia era como que rechazaba mi parte china, de hecho, recuerdo que quería operarme los ojos y además es que tenía una forma de ser muy española.

Eva: Me decían: pues quedamos en tal. [Yo] No, no te puedo decir la hora (...), tengo que decir el mismo día si puedo o no, porque en vez de quedar como el resto, [yo] tenía que ir a trabajar a la tienda [negocio familiar].

10 Pudiendo incluir tanto emociones negativas, como positivas. 
Dandan: nosotros [sus primos/as y ella] teníamos mucha autonomía, más que los niños españoles yo creo, porque ellos no hacen nada, se lo dan todo hecho.

Al hablar de su adolescencia es común entre la muestra -independientemente de su crianza temprana en China o Españala referencia a la diferencia o lo diferente, que tomaba diversas formas en su día a día. En la esfera social, dicha diferencia se asocia directamente con su apariencia física, sus rasgos fenotípicos como marcador que les posiciona como 'los/as otros/as'. En concordancia con las experiencias de otros/as menores descendientes de migrantes (Devine y Macneela, 2008; Pavez Soto, 2012a), todas las personas informantes relatan haber sufrido de actitudes racistas hacia ellas, especialmente durante su adolescencia. Este sentimiento es remarcado por los dos informantes que llegaron a España como adolescentes, en parte, porque su acento es percibido como no completamente español. Esta visibilidad de la diferencia está presente también en los testimonios de los/as informantes de RoblesLlana (2018), quien señala este factor como clave en la construcción identitaria de los/as descendientes de migrantes chinos en España.

Otra referencia común entre los/as informantes hace alusión a la actitud laxa del profesorado ante las actitudes racistas hacia ellos, siendo habitual la recomendación por su parte de ignorar a aquellas personas que les increpaban e incluso, en ocasiones, les agredían físicamente, en lugar de reprobar firmemente sus actitudes y aplicar medidas correctivas. A menudo, el mecanismo de defensa resultante consistía en tratar de pasar lo más desapercibidos posible. Mecanismo que, según diferentes informantes, es el característico del colectivo chino en España. Curiosamente, una menor percepción de la diferencia en el entorno escolar y social más amplio es reportada por aquellas personas que vivían en pueblos o ciudades pequeñas. Asimismo, resulta interesante que los dos informantes dentro de la muestra que no han terminado la educación secundaria ${ }^{11}$ —ambos chicos, de Madrid y Barcelona respectivamente- comparten los mismos elementos en su discurso: el sentimiento de estar fuera de lugar en el instituto, vinculado a su etnicidad, y el ser señalado como diferente; su énfasis en sus buenos resultados académicos y su decisión de abandonar los estudios y comenzar a trabajar. Sin

\footnotetext{
11 Ver Anna Marsden (2015).
} 
embargo, uno de ellos pertenece al grupo de dos informantes que llegaron a España como adolescentes, mientras el otro lo hizo a la edad de 5 años.

La diferencia está presente también en la esfera familiar, al comparar ésta con su entorno: las prácticas, actitudes y comportamiento de sus familias y aquellas de las familias no chinas, principalmente, las españolas. Así las costumbres, derechos y obligaciones a las que los/as informantes hacían frente en sus familias diferían de las de la mayoría de su entorno, en cuestiones como la celebración de ciertas festividades, la ausencia de viajes y periodos vacacionales en familia y, especialmente, hace alusión a un mayor grado de prohibiciones y una menor libertad de movimiento y horarios (Robles-Llana, 2018). A su vez, siendo estos últimos más restrictivos para las chicas que para los chicos, en consonancia con conflictos y disciplina diferencial en base al género (Gardner y Mand, 2012; Foner y Dreby, 2011; Dreby 2010). Sin embargo, era común para ambos la colaboración en el negocio familiar, particularmente durante los fines de semana, disminuyendo su disponibilidad de tiempo de ocio y habiendo de adaptar este a los horarios del negocio.

Por último, la diferencia es subrayada por parte de sus familias. Por un lado, el énfasis en la diferencia de algunos progenitores se basa en su propia experiencia e incorpora un mecanismo protector en aras de preparar a sus hijos/as ante las dificultades que deban afrontar en su condición de descendientes de migrantes. Por otro, las quejas por parte de sus familias hacia a ellos/as por ser 'demasiado españoles' son habituales. Estas se centran en las actitudes, las diferentes nociones de respeto y disciplina, así como los valores esenciales, tales como la importancia de la familia o el trabajo, y otros como el tipo de ocio o comida preferida. Asimismo, el uso del español entre hermanos es percibido como una traba para el mantenimiento de la herencia cultural (Said y Zhou, 2017).

Pese a la preocupación de sus progenitores por lo que éstos perciben como un exceso de españolidad, no es habitual que los/ as informantes fuesen enviados a China a pasar una temporada allí durante su adolescencia, como ocurre en otras familias transnacionales (Foner, 2009a; Orellana et al., 2001; 2001; Soto, 1989). Sin embargo, sí abogaban por el contacto directo con otras personas étnicamente chinas y con China, para trabajar o desarrollar la forma normativa de ser chino/a que sus familias demandan. Principalmente, a través de cursos de idioma chino en escuelas de 
fines de semana y vacaciones de verano en China, ya sea con sus familias o en campamentos de verano destinados a descendientes de migrantes internacionales (Masdeu Torruella, 2019) ${ }^{12}$.

Durante su adolescencia, en torno a la mitad de los/as informantes pasaron uno o varios veranos con sus familias en China, siendo las personas más jóvenes las que reportan un mayor número de visitas, coincidiendo con la progresiva asequibilidad del transporte internacional. Éstas describen dichas experiencias como muy positivas, aunque entendidas en términos vacacionales. En paralelo, y de manera generalizada, desde una edad temprana sus progenitores les han proporcionado películas y series de televisión (formato VCD/ DVD) para que se familiaricen con el idioma mandarín (a menudo en sus casas se habla el dialecto de la zona de origen), así como la historia, cultura y entorno de China.

Existe una percepción compartida por la mayor parte de los/as integrantes de la muestra de una excesiva demanda y presión por parte de sus progenitores, en diversas facetas de su vida familiar y social (Zhou, 2009). Relatan haber sentido presión en el ámbito de los estudios, circunstancia común a otros descendientes de migrantes, que se enfrentan a altas expectativas académicas (Foner y Dreby, 2011; Pavez Soto, 2012a), pese a que sus progenitores no se involucren de manera directa en su vida escolar (Robles-Llana, 2018). Cabe destacar que, a menudo, éstos no disponen de los recursos para hacerlo debido a un escaso dominio del idioma y un generalizado nivel de estudios básicos, como ocurre en este caso. Los/as informantes señalan que dicha presión no es algo que haya condicionado totalmente sus vidas, como puede suceder en el caso de sus familiares y amistades en China, donde el futuro de la familia depende de la trayectoria fructífera de una sola persona descendiente, evocando así una comparativa entre el 'aquí' y el 'allí' (Colombo y Rebughini, 2012). De manera similar a los integrantes del estudio de Tse y Waters (2013) sobre adolescentes Hongkoneses en Canadá, la etapa de la adolescencia para los/as informantes se caracteriza por hacer frente a crecientes responsabilidades, alguno/a de los informantes con una gran autonomía desde edad temprana y, sin embargo, también una creciente presión y control por parte de sus madres y padres.

12 Conocidos como 'Xungen zhi lü' ('寻根之旅') o 'Ruta en búsqueda de raíces', son campamentos que buscan conectar a los/as descendientes de migrantes chinos en el mundo con China y su cultura. 
Entre la muestra, las discrepancias con sus progenitores son generalizadas. Pese a ello, en su mayoría han mostrado obediencia a los mismos, dando lo mejor de sí en el colegio y ayudando en el negocio y en casa, apuntando a la coexistencia con cuidado y cooperación (Foner y Dreby, 2011). Sin embargo, cuatro de las personas entrevistadas - tres chicos y una chica - se refieren a cierto grado de desafío a la autoridad familiar durante la adolescencia tardía, como ha sido documentado en otras familias transnacionales (Artico, 2003; Menjívar, 2006). Ello es especialmente saliente en uno de los dos informantes que llegó a España como adolescente. Tal como sugiere Hondagneu-Sotelo y Ávila (1997) estos deben adaptarse a un nuevo entorno familiar y social, a la par que hacen frente a las convulsiones de la adolescencia. Sin embargo, el otro informante que llegó como adolescente no hace referencia a ninguna conducta diferencial con respecto al resto de la muestra. En su mayoría, desafiaban la autoridad familiar leve y/o indirectamente. Por ejemplo, llegando un poco más tarde de la hora establecida, pero no mucho, callando para evitar confrontar verbalmente a sus progenitores y en general, evitando cualquier tipo de confrontación directa. Ninguno de ellos/as ha reportado comportamientos problemáticos, como conductas violentas o adictivas.

Finalmente, además de la anteriormente mencionada colaboración en el negocio familiar, estos/as jóvenes hacen referencia a la asunción de otras responsabilidades desde la adolescencia temprana, tales como la realización de tareas domésticas - especialmente en el caso de las chicas (Lee y Pacini-Ketchabaw, 2011; Parreñas, 2005; Valenzuela, 1999) — o el cuidado a sus hermanos/as menores. Además, todas las personas entrevistadas reportan haber realizado tareas de traducción y/o interpretación (Bauer, 2016; Menjívar, 2006; Valenzuela, 1999) para sus progenitores y amistades de la familia, ya sea como acompañantes a visitas médicas, gestionando documentos oficiales o acudiendo a las reuniones de madres y padres en el colegio. La información recogida, por tanto, cuestiona la imagen de los/as menores como sujetos pasivos y dependientes y enfatiza sus contribuciones a la familia (Becker, 2007; Pavez Soto, 2012b). Varias de las personas entrevistadas -independientemente de su crianza temprana en España o Chinaidentifican estas como un modo de aprendizaje hacia la madurez, especialmente si se comparan con los/as jóvenes españoles, quienes apuntan, solo piensan en pasárselo bien. Estableciendo así una comparativa entre los valores y actitudes asociados a las sociedades 
donde viven los informantes y aquellas relativas al país de origen de sus familias (Colombo y Rebughini, 2012; Pavez Soto, 2012b).

En resumen, en esta etapa si bien no existe una diferencia generalizada entre las experiencias de aquellas personas cuya infancia temprana transcurrió en China y para aquellas que transcurrió en España, sí que la edad de reunificación en España aparece como factor diferencial, en tanto que condiciona sus experiencias. En concreto, aquellas que llegaron a España como adolescentes reportan una mayor presión social, siendo su acento percibido como no completamente español, propiciando a una articulación de la diferencia en la esfera social más marcada. No obstante, no da lugar a otras diferencias en cuestiones que caracterizan los relatos de los/as informantes con respecto a esta etapa. En cambio, aquellas personas que vivían en pueblos o ciudades más pequeñas —independientemente de haber sido total o parcialmente creados en España- reportan una menor percepción social de diferencia en esta etapa.

\section{Jóvenes adultos (18-31 años)}

Xingfu: ¡hombre, ya que los tengo [hijos/as], los cuido yo!

Feifei: Las madres suelen ser más abiertas y ellos [los padres] más tradicionales.

Wendi: (...) por ejemplo, yo voy a abrazar a mi madre iy ella cree que es raro! como que me empuja, pero es porque yo soy muy expresiva y ellos no son así.

Durante su infancia, más de la mitad de los/as informantes mantuvo una estrecha relación con sus abuelos/as, a nivel físico, emocional y social, ya sea en China o en España. Con el paso de los años, y a menudo mediado por la distancia física, la relación entre nietos/as y quienes fueron sus cuidadores es muy irregular. Mientras algunas de estas personas solo tienen contacto con sus abuelos/as cuando viajan a China o en fechas especiales, otras mantienen un alto grado de interacción (videollamadas, mensajes de voz, mensajes de texto), así como un conocimiento periférico de sus vidas y acciones (Madianou, 2016) mediante el muro de acontecimientos de WeChat ${ }^{13}$. Su generación, la de las abuelas y abuelos, es retratada,

13 Popular red social entre las personas de origen chino. Incorpora servicios de mensajería y llamada de voz y vídeo gratuita, red social con un muro de acontecimientos similar al de Facebook, además de otros servicios como el pago online. 
en términos generales, como flexible, en contraste con el carácter estricto con el que suelen identificar a sus progenitores.

La idea de madres más cercanas y comprensivas frente a padres inflexibles y a menudo descritos como 'tradicionales' (Pavez Soto, 2012) está presente como un elemento común a toda la muestra, aunque los grados de separación entre ambos (madre y padre) difieren. Asimismo, a menudo los/as informantes, tanto quienes pasaron parte de su infancia en China como los/as que no, se posicionan como diferentes dentro de sus familias, a través de expresiones que incorporan la referencia España-China, tales como: "ellos [progenitores] saben que yo soy muy español, piensan que soy una batalla perdida" (Mario, 27 años) "no parezco china" (Wendi, 19 años) o "he crecido aquí [en España], es diferente" (Daquan, 29 años). Cabe entender dichas diferencias y referencias identitarias en el contexto familiar, sin que sean siempre necesariamente extensibles a otros contextos sociales más amplios ${ }^{14}$.

Las personas entrevistadas hacen referencia al apremio por parte de sus progenitores y otros miembros de la familia extensa por encontrarles una pareja y tener descendencia, siendo esta especialmente alta para las chicas. Pese a que ello sucede una vez los/as informantes son ya adultos/as, está en cierto modo enmarcado en el proceso de crecimiento o de hacerse mayor. Esto es, dado que todavía no tienen descendencia, su rol familiar más sobresaliente continúa siendo aquel de hijo/a. Los/as informantes relatan que miembros de la familia extensa, sus padres y, sobre todo sus madres, les proveen de contactos de posibles parejas. Pese a que refieren al afán controlador de sus familias en estos asuntos, en comparación con la libertad de elección y ausencia de presiones que experimentan sus amistades españolas, también subrayan el hecho de que, sin ninguna duda, dicha presión es mucho menor que la soportada por sus familiares y amistades en China, evocando nuevamente la comparativa aquí-allí (Colombo y Rebughini, 2012. Pavez-Soto, 2012b) para hacer referencia a diferentes grados de presión.

Las parejas de origen chino son percibidas como más adecuadas por parte de sus familias, dado que comparten valores culturales e idioma (Foner y Dreby, 2011; Kibria, 1997). Sin embargo, mientras algunos/as consideran que una persona de origen chino criada

14 Ver: Robles, 2018. 
en Europa sería más compatible con su hijo/a, otros prefieren a alguien 'chino, chino', expresión frecuentemente utilizada por los informantes y su generación para referirse a aquellas personas que han sido socializadas en China.

Por su parte, los entrevistados/as conciben a las parejas de origen chino como más dispuestas a aceptar las responsabilidades asociadas a la familia a una edad más temprana que las personas españolas que, en general, son referidas como inmaduras. Esto es especialmente subrayado por los dos informantes que llegaron a España como adolescentes. Sin embargo, en su mayoría, han tenido relaciones tanto como personas de origen chino como español y no cierran la puerta a ninguna de las dos opciones, pese a que la mayoría imagina que, con alguien de familia china criado/a en España, o alguien “... chino como yo. Que no sea chino-chino tampoco, que tengamos un poco en común", como lo describe Eva (21 años), sería más fácil.

Esa mayor facilidad hace referencia a un tipo de vida y forma de pensar similares, resultantes de una socialización —-total o parcialen España, que se vería también reflejada también en el concepto de familia y cuidados. Los/as informantes -independientemente de haber sido total o parcialmente criados en España- construyen sus narrativas de relevo generacional, en su rol como futuros madres y padres, en base a una oposición generacional en cuanto a la gestión de cuidados en la infancia, apuntando a una diferencia entre la forma de pensar y proceder de sus progenitores, en pasado; y la suya propia, como futuro corrector; y vertebrando su discurso en base a sus propias experiencias como menores y a la ideología de familia dominante en España (Lamas Abraira, en revisión). Así, abogan por un reequilibrio entre la esfera familiar y laboral, con un modelo de familia nuclear como base, con interacción diaria y de calidad entre madre, padre e hijos/as, y una relación materno/paternofilial más cercana en la que muestras de afecto entendidas como tal en el contexto español (ej. besos, abrazos, verbalización directa de emociones, ect.) estén presentes, apuntando a una diferente gestión de la crianza (Foner, 2009b). Cabe destacar, sin embargo, que las circunstancias y los recursos — capital social, económico y cultural- difieren ampliamente para ambas generaciones en el mismo estadio vital.

Los términos 'cultura' y 'raíces' son frecuentemente usados por los/as informantes para referirse a la necesidad de enseñar mandarín a su descendencia y lamentan que ellos/as no tengan un 
buen dominio del mismo ${ }^{15}$. Además, alegan razones prácticas, ya que puede resultar muy útil para su futuro laboral. Solo tres de ellos subrayan la necesidad de enseñarles al menos nociones básicas de la lengua o dialecto de la región de origen de su familia, además de mandarín, siendo dos de ellos los informantes que llegaron a España como adolescentes. Por su parte, todas las personas informantes han visitado China al menos una vez siendo ya mayores de edad con una mayor frecuencia para las más jóvenes de entre la muestra, quienes reportan un alto grado de contacto transnacional también a distancia, a través de apps de mensajería y redes sociales, mientras que este está prácticamente ausente en el caso de las más mayores. Asimismo, aquellos/as informantes que no pasaron parte de su infancia en China reportan un menor grado de interacción directa e indirecta (Haikkola, 2011).

Todos los/as informantes se posicionan en tiempo presente y futuro en España y, salvo una excepción que plantea la duda ${ }^{16}$, no piensan en mudarse a China para comenzar una vida allí, ni tampoco en enviar a sus hijos/as durante su infancia. Además, subrayan las dificultades que conllevaría su adaptación a los ritmos y modos de vida en China (Masdeu Torruella, 2014; Robles-Llana, 2018) y el hecho de que allí su acento, manejo del idioma, estética e incluso gestos les evidencian como chinos/as diferentes, debido a su diferente socialización (Sigad y Eisikovits, 2010). Sin embargo, sí consideran estancias en China como una posibilidad a corto o medio plazo, ligado a oportunidades educacionales o laborales (Masdeu Torruella, 2019; Masdeu Torruella y Sáiz López, 2019). Además de dos personas que cursaron estudios de idioma chino en China, tres informantes estudian o han estudiado en territorios o países de mayoría étnica china (Hong Kong, Taiwan y Singapur) ya sea como estudiantes de intercambio o de máster. Otra informante hizo lo propio en Reino Unido. Así, al menos para una parte importante de la muestra, sus experiencias de movilidad distan ampliamente de aquellas de sus progenitores a su edad.

15 Mientras el nivel de expresión oral es muy variable, la mayoría tiene un nivel de comprensión lectora y escritura muy básico o incluso nulo, pese a que algo más la mitad de los informantes acudieron, por periodos de tiempo diferentes, a clases de mandarín en escuelas de fin de semana (en el caso de pueblos o ciudades pequeñas, en aquella época, esta opción no estaba disponible) o en China.

16 Una joven de 22 años parcialmente criada en China. 
Asimismo, los/as informantes rechazan la idea de continuar con el negocio familiar y ambicionan un tipo de vida diferente a la de sus progenitores, con un reequilibrio entre la esfera laboral, familiar y de ocio. Del total de la muestra, solo dos informantes carecen de estudios universitarios - ya sea en curso o finalizados- en contraste con la generación de sus progenitores, quienes, salvo una excepción, disponen de nivel de estudios de primaria o secundaria. Así, abogan por una inserción laboral diferente, con una preferencia por profesiones liberales y el trabajo asalariado y, en menor grado, la inversión de capital económico derivado del negocio familiar en el proyecto empresarial propio de los descendientes (Yiu, 2013) ${ }^{17}$. Siendo sus ideales de futuro construidos en base al proceso de movilidad social ascendente experimentado por sus familias a raíz del proyecto migratorio (Masdeu Torruella y Sáiz López, 2019).

En resumen, en lo referente a esta etapa, una vez más, la edad de reunificación en España para aquellas personas parcialmente criadas en China aparece como factor diferencial. Así, los únicos dos informantes entre la muestra que pasaron el total de su infancia en China, mudándose a España como adolescentes, muestran una preferencia por parejas socializadas en China e interés por enseñar a sus descendientes el idioma o dialecto familiar, además del mandarín, mostrando una mayor inclinación hacia China o lo chino, pese a que sus preferencias en otras cuestiones no difieren con respecto al resto de la muestra.

Por otro lado, aquellas personas parcialmente criadas en China, independientemente de la edad de reunificación en España, muestran - en términos generales- un mayor grado de relación con el área de origen de su familia en China. No obstante, no siempre es el caso. Además, tampoco se da una relación directa, ya que la edad de los informantes constituye un factor clave en la articulación de estas relaciones, y en general, una mayor edad en tiempo presente (aprox. 23-30 años) conlleva un menor nivel de contacto con China a lo largo de su trayectoria vital, dada la imposibilidad de mantener contacto frecuente y continuado - debido a las limitaciones en el

17 Las expectativas y potencial de inserción laboral no siempre se corresponden con la realidad tangible y, a menudo, los/as descendientes de migrantes chinos en España encuentran en los nichos étnicos y en el proyecto empresarial (ver Yiu, 2013) una alternativa laboral viable frente a la crisis de desempleo juvenil generalizada en el país. 
transporte y comunicaciones a distancia- en etapas anteriores. Sin embargo, aquellas personas más jóvenes (aprox. 18-22 años) han podido disfrutar de las ventajas de un mundo interconectado desde una edad temprana, favoreciendo la continuidad en sus relaciones transnacionales.

\section{CONCLUSIONES}

El flujo migratorio continuado y cuantitativamente significativo de China a España tiene una historia corta, de manera generalizada no ha sido sino recientemente que, como generación, los/as descendientes de migrantes chinos en España han comenzado a alcanzar la mayoría de edad y su condición actual como jóvenes adultos/as. Dos constantes, su estatus de descendientes de migrantes chinos en España y una diferente socialización - ya sea total o parcial en España- con respecto a la socialización en China de sus familias, modelan sus trayectorias y vertebran sus relatos, condicionando una experiencia generacional específica (Colombo y Rebughini, 2012; Elder, 1998; Nyiri, 2014) que oscurece o sobrepasa las peculiaridades resultantes de los diferentes itinerarios que caracterizan su infancia temprana (en China o España).

La noción de diferencia aparece como transversal en sus relatos a través de las diversas etapas vitales, en distintas esferas y variadas formulaciones. Cabe alejar el concepto de la diferencia como algo negativo, dependiendo este de las circunstancias en las que su percepción, propia o ajena, se inscribe. Asimismo, el concepto de diferencia no es necesariamente constante ni estable, sino cambiante, fluido y permeable en el tiempo. En el contexto de su experiencia generacional específica, las comparativas entre lo 'español' y lo 'chino' —que incluye, pero que no se limita al 'aquí' y 'allí'- son constantes. Sin embargo, ello no condiciona un (auto) posicionamiento estático de los/as informantes a uno u otro lado, pivotando entre ambos y sus múltiples articulaciones.

Aunque las hipótesis previas al campo, así como estudios similares en otro contextos, apuntaban a unas experiencias claramente diferenciadas para aquellas personas que habían sido parcialmente criadas en China y aquellas que no, como categorías infranqueables, que conllevaban una etiqueta determinada e 
ineludible, con consecuencias extendidas en el tiempo como huellas que prevalecen a través de sus trayectorias vitales, estas se muestran más permeables y con un alcance mucho más limitado al esperado.

Esta condición relativa y relacional, sin embargo, se torna estática y frontal en la comparativa entre la generación la de sus progenitores y la suya propia. Pese al énfasis en la diferencia generacional, cabe destacar la noción de vidas 'vinculadas' o 'interconectadas' (linked lives) (Elder, 1998) que sugiere que las vidas y trayectorias de los individuos se ven afectadas o condicionadas por aquellas de otras personas cercanas, ocupando la familia un lugar central. En los relatos en primera persona de los/as informantes, se hace referencia a estos vínculos, permitiendo rastrear los lazos y uniones entre las trayectorias de ambas generaciones, entre sus experiencias, sus oportunidades y las formas de ser resultantes. Siendo la familia, y su cuidado, decisivos en el desarrollo de su experiencia generacional específica (Lamas Abraira en revisión).

En este artículo, la exploración de las trayectorias de los/ as descendientes de migrantes chinos en España a través de una aproximación de curso vital evita la fragmentación y descontextualización de sus vivencias y aporta una visión holística de las mismas, posibilitando el rastreo de interconexiones entre las diversas experiencias, etapas vitales, personas y tiempos (pasado, presente y futuro). Dado lo reducido de la muestra, los resultados no son generalizables. Sin embargo, se pone de manifiesto la importancia ineludible del contexto y la conveniencia de abogar por estudios que aporten una visión no fragmentada de las trayectorias de los descendientes de familias migrantes y sus interconexiones.

\section{REFERENCIAS BIBLIOGRÁFICAS}

Antman, F. M. (2012). The Impact of Migration on Family Left Behind. En A. F. Constant, K. F. Zimmermann (eds.), International Handbook on the Economics of Migration. Cheltenham y Northampton: Edward Elgar.

Artico, C. I. (2003). Latino Families Broken by Immigration: The Adolescent's Perceptions. New York: LFB Scholarly Publishing.

Bartley y Spoonley (2008). Intergenerational Transnationalism: 1.5 Generation Asian Migrants in New Zealand. International Migration, 46(4), 63-84. doi:10.1111/j.1468-2435.2008.00472.x 
Bauer, E. (2016). Practising Kinship Care: Children as Language Brokers in Migrant Families. Childhood, 23(1), 22-36. https://doi. org/10.1177/0907568215574917

Becker, S. (2007). Global Perspectives on Children's Unpaid Caregiving in the Family: Research and Policy on 'Young Carers' in the UK, Australia, the USA and SubSaharan Africa. Global Social Policy, 7(1), 23-50. https:// doi.org/10.1177/1468018107073892

Beltrán Antolín, J. (2003). Los ocho inmortales cruzan el mar. Chinos en Extremo Occidente. Barcelona: Edicions Bellaterra.

Beltrán Antolín, J. (2006). El empresariado como modo de vida. El caso de los inmigrantes chinos. En Empresariado étnico en españa. parte iv: capital social y cultural. 231-248. https://www.cidob.org/es/publicaciones/serie_de_ publicacion/monografias/monografias/empresariado_etnico_en_espana

Biao, X. (2007). How Far are the Left-behind Left Behind? A Preliminary Study in Rural China. Population, Space and Place, 13(3), 179-191. https://doi.org/10.1002/psp.437

Bohr, Y. y Tse, C. (2009). Satellite Babies in Transnational Families: A study of Parents' Decision to Separate from their Infants. Infant Mental Health Journal, 30, 265-286. doi: 10.1002/imhj.20214

Bonizzoni, P. (2015). Here or There? Shifting Meanings and Practices in Mother-Child Relationships across Time and Space. International Migrations, 53, 166-182. https://doi.org/10.1111/imig.12028

Braun, V. y Clarke, V. (2012). Thematic analysis. En Cooper, H. (Ed.), APA handbook of research methods in psychology: Vol. 2. Research designs (pp. 57-91). Washington, DC: American Psychological Association.

Buriel, R. (1993). Childrearing orientations in Mexican American families: The influence of generation and sociocultural factors. Journal of Marriage and the Family, 55(4), 987-987. https://doi.org/10.2307/352778

Chang, H., Dong, X. y MacPhail, F. (2011). Labor Migration and Time Use Patterns of the Left-behind Children and Elderly in Rural China. World Development, 39(12), 2199-2210. https://doi.org/10.1016/j. worlddev.2011.05.021

Chen, F., Liu, G. y Mair, C. A. (2011). Intergenerational Ties in Context: Grandparents Caring for Grandchildren in China. Social forces, 90(2), 571-594. doi: $10.1093 / \mathrm{sf} / \mathrm{sor} 012$

Colombo, E. y Rebughini, P. (2012). Children of Immigrants in a Globalized World. A Generational Experience. Houndmills: Palgrave Macmillan.

Da, W.W. (2003). Transnational Grandparenting: Child Care Arrangements among Migrants from the People's Republic of China to Australia. Journal of International Migration and Integration, 4(6),79-103. https://oi. org/10.1007/s12134-003-1020-4

Devine, D., Kenny, M. y Macneela, E. (2008). Naming the 'Other': Children's Construction and Experience of Racisms in Irish Primary Schools. Race Ethnicity and Education, 11(4), 369-385. Doi: 10.1080/13613320802478879 
Dobson, M. E. (2009). Unpacking Children in Migration Research. Children's Geographies, 7(3), 355-360. Doi: 10.1080/14733280903024514

Dreby, J. (2007). Children and Power in Mexican Transnational Families. Journal of Marriage and Family, 69(4), 1050-1064. www.jstor.org/stable/4622507

Dreby, J. (2010). Divided by Borders: Mexican Migrants and their Children. Berkeley: University of California Press

Elder, G. H. (1998). The Life Course as Developmental Theory. Child Development, 69(1), 1-12. https://doi.org/10.1111/j.1467-8624.1998.tb06128.x

Foner y Dreby (2011). Relations Between the Generations in Immigrant Families. Annual Review of Sociology, 37, 545-564. https://www.annualreviews.org/doi/abs/10.1146/annurev-soc-081309-150030

Foner, N. (2009 a). Introduction: Intergenerational Relations in Immigrant Families. In N. Foner (ed.), Across Generations: Immigrant Families in America (pp. 1-20). NewYork: New York University Press.

Foner, N. (2009 b). Mobility Trajectories and Family Dynamics: History and Generation in the New York Immigrant Experience. Diaspora, 18(1/2), 24-43. https://www.muse.jhu.edu/article/573612.

Fresnoza-Flot, A. (2018). "Rethinking Children's Place(s) in Transnational Families: Mobile Childhoods in Filipino International Migration”, Childhood and Parenting in Transnational Settings,.

Gardner, K. (2012). Transnational Migration and the Study of Children: An Introduction. Journal of Ethnic and Migration Studies, 38(6), 889-912. https://doi.org/10.1080/1369183X.2012.677170

Gardner, K. y Mand, K. (2012). 'My Away is Here': Place, Emplacement and Mobility amongst British Bengali Children. Journal of Ethnic and Migration Studies, 38(6), 969-986. https://doi.org/10.1080/136918 3X.2012.677177

Gaspar, S. (2019). Chinese descendants' professional pathways: moving to new businesses?. Portuguese Journal of Social Science, 18(1),91-108. https://doi.org/10.1386/pjss.18.1.91_1

Guang, Y., Feng, Z., Yang, G., Yang, Y., Wang, L., Dai, Q., ... Zhao, M. (2017). Depressive Symptoms and Negative Life Events: What Psycho-social Factors Protect or Harm Left-behind Children in China?. BMC Psychiatry, 17(1), 402. doi: 10.1186/s12888-017-1554-1

Haikkola, L. (2011). Making Connections: Second-Generation Children and the Transnational Field of Relations. Journal of Ethnic and Migration Studies, (37)8, 1201-1217. https://doi.org/10.1080/1369183X. 2011.590925

Hoang, L. A., Lam, T., Yeoh, B. S. A. y Graham, E. (2015). Transnational Migration, Changing Care Arrangements and Left-behind Children's Responses in South-east Asia. Children's Geographies, 13(3), 263-277. doi: 10.1080/14733285.2015.972653

Hondagneu-Sotelo, P. y Ávila, E. (1997). “I'm Here, but I'm There”: The Meanings of Latina Transnational Motherhood. Gender and Society, 11(5), 548-571. https://doi.org/10.1177/089124397011005003 
Kibria, N. (1997) The construction of 'Asian American': Reflections on intermarriage and ethnic identity among second-generation Chinese and Korean Americans. Ethnic and Racial Studies, (20)3, 523-544. https://doi. org/10.1080/01419870.1997.9993973

Kim, N. (2009). Finding Our Way Home: Korean Americans, Homelands Trips, and Cultural Foreignness.' in Tsuda, T.(ed), Diasporic Homecomings: Ethnic Return Migrants in Comparative Perspective (pp. 305-24). Stanford: Stanford University Press.

Lamas Abraira, L. (2021). El cuidado en las familias transnacionales qingtianesas. En J. Beltrán Antolín (ed.), Paradigmas emergentes de Asia Oriental (pp. 161-182). Barcelona: Edicions Bellaterra.

Lamas Abraira, L. (en revisión). The Corrective Future: Contextualizing Chinese Migrants' Descendants Life Paths and Family-Care Ideals. Journal of Youth Studies.

Lee, J. y Pacini-Ketchabaw, V. (2011). Immigrant Girls as Caregivers to Younger Siblings: a Transnational Feminist Analysis, Gender and Education, 23(2),105-119. https://doi.org/10.1080/09540251003674063

Lei, L., Liu, F. y Hill (2017). Labour Migration and Health of Left-Behind Children in China. The Journal of Development Studies, 54(1), 93-110. https://doi.org/10.1080/00220388.2017.1283015

Leinaweaver, J. B. (2007), On Moving Children: The Social Implications of Andean Child Circulation. American Ethnologist, 34(1), 163-180. https:// doi.org/10.1525/ae.2007.34.1.163

Levitt, P. (2009). Roots and Routes: Understanding the lives of the second generation transnationally. Journal of Ethnic and Migration Studies, 35(7), 1225-1242. https://doi.org/10.1111/imre.12314

Lie, M.L.S. (2010). Across the Oceans: Childcare and Grandparenting in UK Chinese and Bangladeshi Households. Journal of Ethnic and Migration Studies, 36(9), 1425-1443. https://doi.org/10.1080/1369183X.2010.491746

Louie, V. (2006). Growing up Ethnic in Transnational Worlds: Identities among Second-generation Chinese and Dominicans. Identities, 13(3), 363-394. https://doi.org/10.1080/10702890600838118

Lu, Y. (2012). Education of Children Left Behind in Rural China. Journal of Marriage and Family, 74(2), 328-341. https://doi.org/10.1111/j.17413737.2011.00951.x

Madianou, M. (2016). Ambient Co-presence: Transnational Family Practices in Polymedia Environments. Global Networks, 16(2), 183-201. https:// doi.org/10.1111/glob.12105

Marsden, A. (2015). Second Generation Chinese and New Processes of Social Integration in Italy. En In: Baldassar L., Johanson G., McAuliffe N., Bressan M. (eds), Chinese Migration to Europe. Londres: Palgrave Macmillan.

Masdeu Torruella, I. (2014). Mobilities and Embodied Transnational Practices: An Ethnography of Return(s) and other Intersections between China and Spain (Tesis doctoral). Barcelona: Universidad Autònoma de Barcelona. 
Masdeu Torruella, I. (2019). Migrants' Descendants and New Mobilities between China and Spain. International Migration. https://doi.org/10.1111/ imig. 12619

Masdeu Torruella, I. y Sáiz Lopez, A. (2019). Transiting (in) Shanghai: Highskilled professionals from Spain to China. Transitions: Journal of Transient Migration, 3(1), 59-70. https://doi.org/10.1386/tjtm.3.1.59_1

Menjívar, C. (2006). Family Reorganization in a Context of Legal Uncertainty: Guatemalan and Salvadoran Immigrants in the United States. International Journal of Sociology of the Family, 32(2), 223-244. https://www. jstor.org/stable/23030196

Morooka, H., \& Liang, Z. (2009). International Migration and Education of Left Behind Children in Fujian, China. Asian and Pacific Migration Journal, 18(3), 345-370. https://doi.org/10.1177/011719680901800302

Neysmith, S. M. y Zhou, Y. R. (2013). Mapping another dimension on a feminist ethics of care:Family-based transnational care. The International Journal of Feminist Approaches to Bioethics, 6(2), 141-159. https://doi. org/10.3138/ijfab.6.2.141

Ní Laoire, C. (2011). Narratives of 'Innocent Irish Childhoods': Return Migration and Intergenerational Family Dynamics. Journal of Ethnic and Migration Studies, 37(8), 1253-1271. https://doi.org/10.1080/136918 3X.2011.590928

Nieto, G. (2007). La inmigración china en España: una comunidad ligada a su nación. Madrid: Los libros de la catarata.

Olwig, K. F. (2002). A Respectable Livelihood: Mobility and Identity in a Caribbean Family. In N.N. Sorensen \& K. Fog Olwig (eds), Work and Migration: Life and Livelihoods in a Globalized World (pp. 85-105). New York: Routledge.

Orellana, M. F., Thorne, B., Chee, A., \& Lam, W. S. E. (2001). Transnational childhoods: The participation of children in processes of family migration. Social Problems, 48(4), 572-591. https://doi.org/10.1525/sp.2001.48.4.572

Nyíri, P. (2014).Training for transnationalism: Chinese children in Hungary. Ethnic and Racial Studies, 37(7), 1253-1263. https://doi.org/10.1080/014 19870.2014.878029

Parreñas, R. S. (2005). Children of Global Migration. Transnational Families and Gendered Woes. Stanford: Stanford University Press.

Pavez Soto, I. (2012 a). Inmigración y Racismo: Experiencias de la niñez peruana en Santiago de Chile. Si Somos Americanos, 12(1), 75-99. https:// doi.org/10.4067/S0719-09482012000100004

Pavez Soto, I. (2012 b). Sociología de la Infancia: las niñas y los niños como actores sociales. Revista de Sociología, 27, 81-102. Doi: 10.5354/0719529X.2012.27479

Perreira, K. M. y Ornelas, I. J. (2011). The Physical and Psychological WellBeing of Immigrant Children. Inmigrant Children, 21(1), 195-219. DOI:

Phoenix y Bauer (2012). Challenging Gender Practices: Intersectional Narratives of Sibling Relations and Parent-child Engagements in Transnational 
Serial Migration. European Journal of Women's Studies, 19(4), 490-504. https://doi.org/10.1177/1350506812455994

Phoenix, A. y Seu, B. (2013). Negotiating Daughterhood and Strangerhood: Retrospective Accounts of Serial Migration. Feminism \& Psychology, 23(3), 299-316.

Portes A. y Rumbaut R. (2001). Legacies: The Story of the Immigrant Second Generation. Berkeley, CA: University of California Press.

Portes, A. (1997). Immigration Theory for a New Century: Some Problems and Opportunities. The International Migration Review, 31(4), 799-825. https://doi.org/10.2307/2547415

Portes, A., Aparicio, R., Haller, W.(2018). Hacerse adulto en España: la integración de los hijos de inmigrantes. Anuario CIDOB de la Inmigración, 2018,148-81. https://doi.org/10.24241/AnuarioCIDOBInmi.2018.148

Punch, S. (2015). Youth Transitions and Migration: Negotiated and Constrained Interdependencies within and across Generations. Journal of Youth Studies, 18(2), 262-276.

Raffaetà, R.; Baldassar, L. y Harris, A. (2015). Chinese Immigrant Youth Identities and Belonging in Prato, Italy: Exploring the Intersections between Migration and Youth Studies. Identities, 23(4), 422-437.

Robles-Llana, P. (2018). Cultural Identities of Children of Chinese Migrants in Spain: A Critical Evaluation of the Category 1.5 Generation. Identity. https://doi.org/10.1080/15283488.2018.1447481

Said, F. y Zhu, H. (2017). No, no Maama! Say 'Shaatir ya Ouledee Shaatir'!" Children's agency in language use and socialisation.InternationalJournalof Bilingualism, 23(3), 771-785. https://doi.org/10.1177/1367006916684919

Sáiz López, A. (2005). La migración china en España. Características generales. Revista CIDOB d'Afers Internacionals, 68, 151-163. www.jstor.org/ stable/40586129

Sáiz López, A. (2012). Transnationalism, Motherhood, and Entrepreneurship: Chinese Women in Spain. Advances in Gender Research,16, 39-59. https://doi.org/10.1108

Sigad, L. I. y Eisikovits, A. R. (2010). 'You Can't Exactly Act American Here in Israel!': Identity Negotiations of Transnational North American-Israeli Children and Youth. Journal of Ethnic and Migration Studies, 36(7), 1013-1031. https://doi.org/10.1080/1369183X.2010.481590

Soto, I. M. (1989). West Indian Child Fostering: Its Role in Migrant Exchanges. Center for Migration Studies, special issues, 7, 121-137. https://doi. org/10.1111/j.2050-411X.1989.tb00982.x

Stella S. (2018). Towards Transnational Gender Identity. A Case Study of Polish Children Growing up in Norway. Gender, Place \& Culture, 25(1), 134-148. https://doi.org/10.1080/0966369X.2017.1379953

Suârez-Orozco, C., Todorova, I. L. G. y Louie, J. (2002). Making Up for Lost Time: The Experience of Separation and Reunification among Immigrant Families. Family Process, 41(4), 625-643. https://doi.org/10.1111/ j.1545-5300.2002.00625.x 
Suárez-Orozco, C., Bang, H. J. y Ha Y. K. (2012). I Felt Like My Heart Was Staying Behind: Psychological Implications of Family Separations \& Reunifications for Immigrant Youth. Journal of Adolescent Research, 26(2), 222-257. https://doi.org/10.1177/0743558410376830

Tienda, M. y Haskins, R. (2011). Immigrant Children: Introducing the Issue. Inmigrant Children, 21(1), 3-18. https://doi.org/10.1353/foc.2011.0010

Tse, J. K. H. y Waters, J. L. (2013). Transnational Youth Transitions: Becoming Adults between Vancouver and Hong Kong. Global Networks, 13, 535-550. https://doi.org/10.1111/glob.12014

Tymczuk, A (2011). Social Orphans and Care at a Distance: Popular Representations of Childhood in Ukrainian Transnational Families. Global Studies of Childhood, 1(4), 377-387. https://doi.org/10.2304/gsch.2011.1.4.377

Valenzuela, A. (1999). Gender Roles and Settlement Activities among Children and Their Immigrant Families. American Behavioral Scientist, 42(4), 720-742. https://doi.org/10.1177/0002764299042004009

Wei, L. (2011). Moment Analysis and Translanguaging Space: Discursive Construction of Identities by Multilingual Chinese youth in Britain. Journal of Pragmatics, 43(5), 1222-1235. https://doi.org/10.1016/j.pragma.2010.07.035

Wen, M. y Lin, D, (2012). Child Development in Rural China: Children Left Behind by their Migrant Parents and Children of Non-migrant Families. Child Development, 83(1), 120-136. Doi: 10.1111/j.14678624.2011.01698.x

White, A., Ní Laoire, C., Tyrrell, N. y Carpena-Méndez, F. (2011). Children's Roles in Transnational Migration. Journal of Ethnic and Migration Studies, 37(8), 1159-1170. https://doi.org/10.1080/1369183X.2011.590635

Wong, K. R. (2015). The Fengsu-Driven Practice of Sending Infants to China: The Experiences of Chinese Immigrant Mothers in New York. CUNY Academic Works. http://academicworks.cuny.edu/gc_etds/1190

Wu, C. y Chao, R. K. (2011). Intergenerational Cultural Dissonance in Parent-Adolescent Relationships among Chinese and European Americans. Developmental Psychology, 47(2), 493-508. doi: 10.1037/a0021063. PMID: 21219066.

Yiu, J. (2013). Calibrated Ambitions: Low Educational Ambition as a Form of Strategic Adaptation among Chinese Youth in Spain. International Migration Review, 47(3), 573-611. https://doi.org/10.1111/imre.12037

Zhou, M. (2009). Conflict, Coping, and Reconciliation: Intergenerational Relations in Chinese Immigrant Families. In N. Foner (ed.), Across Generations: Immigrant Families in America (pp. 21-46). New York: NYU Press.

Zhou, Y. R. (2013). Toward Transnational Care Interdependence: Rethinking the Relationships between Care. Immigration and Social Policy Global Social Policy, 13(3), 280-298. https://doi.org/10.1177/1468018113499573

Zhou, M. (1997). Segmented Assimilation: Issues, Controversies, and Recent Research on the New Second Generation.The International Migration Review, 31(4), ¡975-1008. doi:10.2307/2547421 